\title{
Microwave sintering of yttria-containing tetragonal zirconia polycrystal (Y-TZP) ceramics
}

\author{
D S PATIL* and B C MUTSUDDY \\ Institute of Materials Processing, Michigan Technological University, Houghton, Michigan \\ 49931, USA \\ * Present address: Laser and Plasma Technology Division, Bhabha Atomic Research Centre, \\ Bombay 400085 , India \\ MS received 16 June 1994; revised 24 August 1994

\begin{abstract}
Sintering of yttria-containing tetragonal zirconia polycrystal (Y-TZP) ceramics was performed in a single-mode cylindrical cavity applicator CMPR-250 operating at $2.45 \mathrm{GHz}$ in the $\mathrm{TM}_{012}$ mode. High heating rates at low power levels were achieved. Rapid heating and cooling resulted in a fine-grain microstructure.

High-purity submicron Y-TZP powders were sintered from an initial green density of $60^{\circ}$ o to final sintered density close to the theoretical density. Microwave sintering offers potential for improving microstructural properties through controlled development of uniform microstructure.
\end{abstract}

Keywords. Microwave sintering; Y-TZP; single-mode applicator.

\section{Introduction}

Tetragonal zirconia polycrystal (TZP) ceramics are one of the most promising materials because of their excellent thermal and mechanical properties. Yttriacontaining tetragonal zirconia (Y-TZP) ceramics consist of tetragonal zirconia $\left(\mathrm{t}-\mathrm{ZrO}_{2}\right)$ grains containing around $2-3 \mathrm{~mol}_{\%} \mathrm{Y}_{2} \mathrm{O}_{3}$ in solid solution. These ceramics are useful as adiabatic engine components in the form of ceramic-metal composite system for thermal insulation. Their low thermal conductivity and high thermal expansion reduce the difficulties encountered with ceramic-metal attachment. The electrical conductivity of TZP ceramics is ionic, which remains reasonably constant for several cycles and for reasonable length of time (Yamamoto et al 1990) and hence they are attractive as an electrolyte for solid oxide fuel cell applications in the temperature range $900-1000^{\circ} \mathrm{C}$. They have also impressive mechanical properties and hence are used as structural material. All these applications require high level of densification in a sintered body.

The toughness of these materials is due to the tetragonal-to-monoclinic transformation which occurs in the process zone around the advancing crack front (Porter et al 1979). Stabilization of zirconia in the tetragonal phase is, therefore, critical. However, to retain the metastable tetragonal $\mathrm{ZrO}_{2}$, it is essential that $\mathrm{ZrO}_{2}$ grain size be less than some critical size which is reported to be in the range of 0.5 to $0.8 \mu \mathrm{m}$ (Lange and Green 1981; Green 1982; Garvie 1984). Above this critical size, the $\mathrm{ZrO}_{2}$ grains transform to the monoclinic form.

The high temperatures which are required for the densification during conventional sintering processes usually result in significant grain coarsening and growth of $\mathrm{ZrO}_{2}$ particles above the critical size. Therefore, it is difficult to retain the desired zirconia phase form. To meet these requirements, numerous processing approaches have been investigated to achieve lower densification temperature. 
The application of microwave processing in ceramics has become an area of intense interest as the realization of the technique's utility has increased. Microwave heating is fundamentally different from conventional heating processes as heat is generated within the material. Microwave energy is deposited instantaneously and volumetrically within the bulk of the ceramic. Thermal gradients and heat flow are in reverse direction in comparison to conventional heating. This, coupled with high heating rate, allows uniform pore elimination and ultrafine microstructure. An excellent review on ceramic processing by microwaves is given by Sutton (1989).

Good uniformity in microwave sintering requires a knowledge of the hightemperature microwave properties of the material as well as the ability to modify and control them.

The difficulties encountered in efficient microwave sintering of ceramics can be reduced by using a resonant cavity. Single-mode resonant systems can be designed to yield very high electric field strength which, in turn, results in high heating rates. The field inside the cavity can be considerably larger than the field outside. This is due to an increase in the quality factor of the cavity (Asmussen et al 1987). Power absorbed by the material being proportional to its dielectric loss factor and the square of the electric field strength (Chabinsky and Eves 1986; Tinga 1986), the use of singlemode applicator definitely helps in putting more power even in materials with low dielectric loss.

Practical-size samples of $\mathrm{Al}_{2} \mathrm{O}_{3}$ and $\mathrm{Al}_{2} \mathrm{O}_{3}: \mathrm{ZrO}_{2}$ were successfully sintered by using single-mode applicator and the results are published in detail elsewhere (Patil et al $1991 \mathrm{a}, \mathrm{b})$.

The objective of this study was to show the feasibility of using a single-mode applicator for microwave sintering of the Y-TZP ceramics.

\section{Experimental set-up}

The experimental system consists of a variable-power $\mathrm{CW}$ microwave power supply ( $3 \mathrm{~kW}$ operating at $2.45 \mathrm{GHz}$ ), two circulators and matched dummy loads, WR-284 wave guide network, forward and reflected power meters (which measure the incident power $P_{\mathrm{i}}$ and the reflected power $P_{\mathrm{r}}$ ), a coaxial input coupling system, Accufibre temperature measurement system (Model $100 \mathrm{C}$, Accufibre, Inc., Beaverton, OR) and Wavemat cavity CMPR-250 (Wavemat Inc., Plymouth, MI). This particular cavity has the ability to focus and match the incident microwave energy into the process material. It can be internally tuned. The details are given elsewhere (Patil et al 1991a).

\section{Sample preparation and experiment}

We have used two types of powders for this study. The first powder TZP-O was as received from Ferro (NZP-A3Y, ARP 5863; Ferro Corporation, Cleveland, OH). The vendor's data show that the average zirconia grain size is $0.274 \mu \mathrm{m}$ and yttria content $3 \mathrm{~mol} \%$. The other powder TZP-CHEM was made by a chemical route. The preparation procedure is as follows. Aqueous solutions of appropriate amounts of $\mathrm{ZrOCl}_{2} \cdot 8 \mathrm{H}_{2} \mathrm{O}$ and $\mathrm{Y}\left(\mathrm{NO}_{3}\right)_{3} \cdot 6 \mathrm{H}_{2} \mathrm{O}$ (Johnson Matthey, Alfa Products, Ward Hill, MA) were mixed to get the desired composition with $\mathrm{Y}_{2} \mathrm{O}_{3}$ as $2.5 \mathrm{~mol} \%$. After homogeneous mixing, water was evaporated slowly and thoroughly. The dried mix was calcined at $1100^{\circ} \mathrm{C}$ 
for $2 \mathrm{~h}$ at a heating/cooling rate of $6^{\circ} \mathrm{C} / \mathrm{min}$. The calcined powder was washed with deionized water to make it free of $\mathrm{Cl}^{-}$radicals. The washed powder was dried under an infrared lamp. The washed and dried powder was milled with isopropyl alcohol using zirconia media for $50 \mathrm{~h}$. The slurry was dried under an infrared lamp and the dried powder was sieved through a 100 mesh screen. The average zirconia grain size as seen under the JEOL JSM-820 scanning electron microscope (JEOL USA, Inc., Peabody, MA) was around $0 \cdot 3 \mu \mathrm{m}$.

These powders were isostatically cold-pressed into cylindrical pellets of approximate size $25.4 \mathrm{~mm}$ dia. $\times 25.4 \mathrm{~mm}$ height at a pressure of $276 \mathrm{MPa}$. The pellets were kept at $100^{\circ} \mathrm{C}$ in an oven for $12 \mathrm{~h}$ to remove moisture. The green densities of the pellets were around $60 \%$ of the theoretical density.

The pellet was embedded in alumina fibre insulation. The insulated pellet was kept in the centre of the base plate of the cavity on a boron nitride platform. The incident microwave power was coupled with the help of the coupling probe. A hole of approximately $3 \mathrm{~mm}$ was drilled through the insulation to view the sample surface by the Accufibre optical pyrometer system through a non-radiating opening on the wall of the cavity. The samples were processed in the cavity according to the heating schedule shown in figure 1 . The temperature shown here is the surface temperature of the sample. It was essential to control the heating and cooling rates to get crack-free samples. The total microwave power (forward and reflected power together) did not exceed $1 \mathrm{~kW}$. The densities of the samples were measured by Archimedes principle. The fractured surfaces of the samples were seen under SEM.

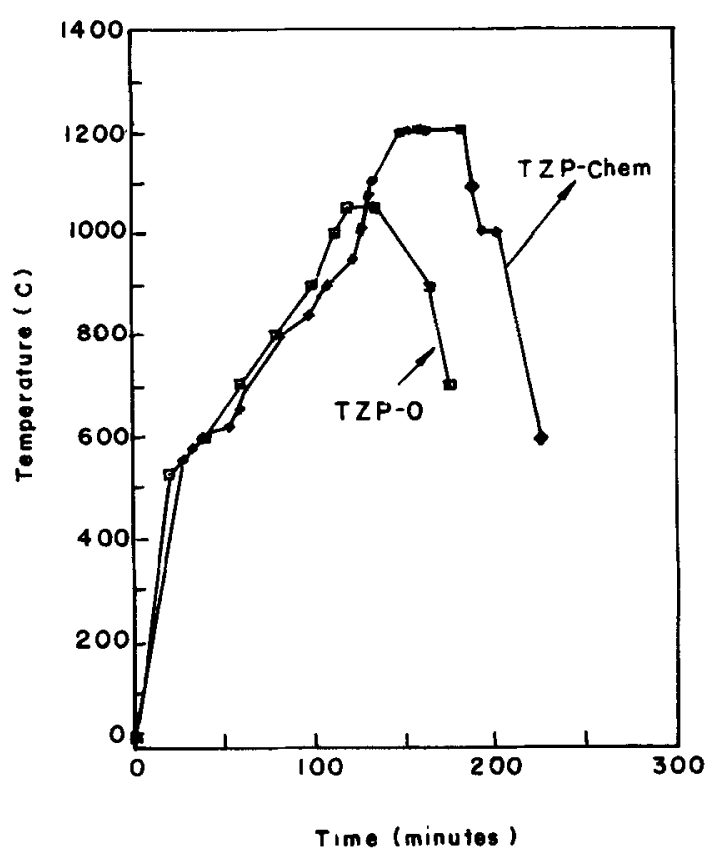

Figure 1. Microwave sintering schedule for TZP samples. 


\section{Results and discussion}

The density measurement on the samples show that the sample TZP-O was sintered to $96.85 \%$ of the theoretical density in $3.16 \mathrm{~h}$ (this includes heating, cooling and hold time at high temperature) and the sample TZP-CHEM was sintered to theoretical density in $4 \mathrm{~h}$.

The sintering consisted of two steps: preheating and sintering stage. The sample was initially heated for a few min at a constant low power. Attempts to reach the final sintering temperature without preheating, i.e. by raising the input power, gave rise to hot spot formation, arcing and eventual cracking of the samples. To achieve sintering uniformity it was necessary to insulate the sample. The gradual increase in power is also essential to get proper densification. With careful control of these conditions thermal runaway which is generally observed in the microwave sintering experiments has been avoided. The densification of the sample was noted by an increase in the reflected power, as the sample fails to absorb more power after densification and hence the reflected power increases. Controlled cooling was essential to avoid cracking of the sample due to thermal shock.

The microstructures/fractured surfaces of the samples as seen under SEM are shown in figures 2 and 3. Fine and uniform microstructure is seen. Microwave sintering should help in retaining zirconia in the metastable tetragonal phase because the sample passes more rapidly through the lower-temperature regime where surface diffusion is relatively more important and the fine-grain microstructure is raised to high temperature where grain boundary and lattice diffusion dominate over surface

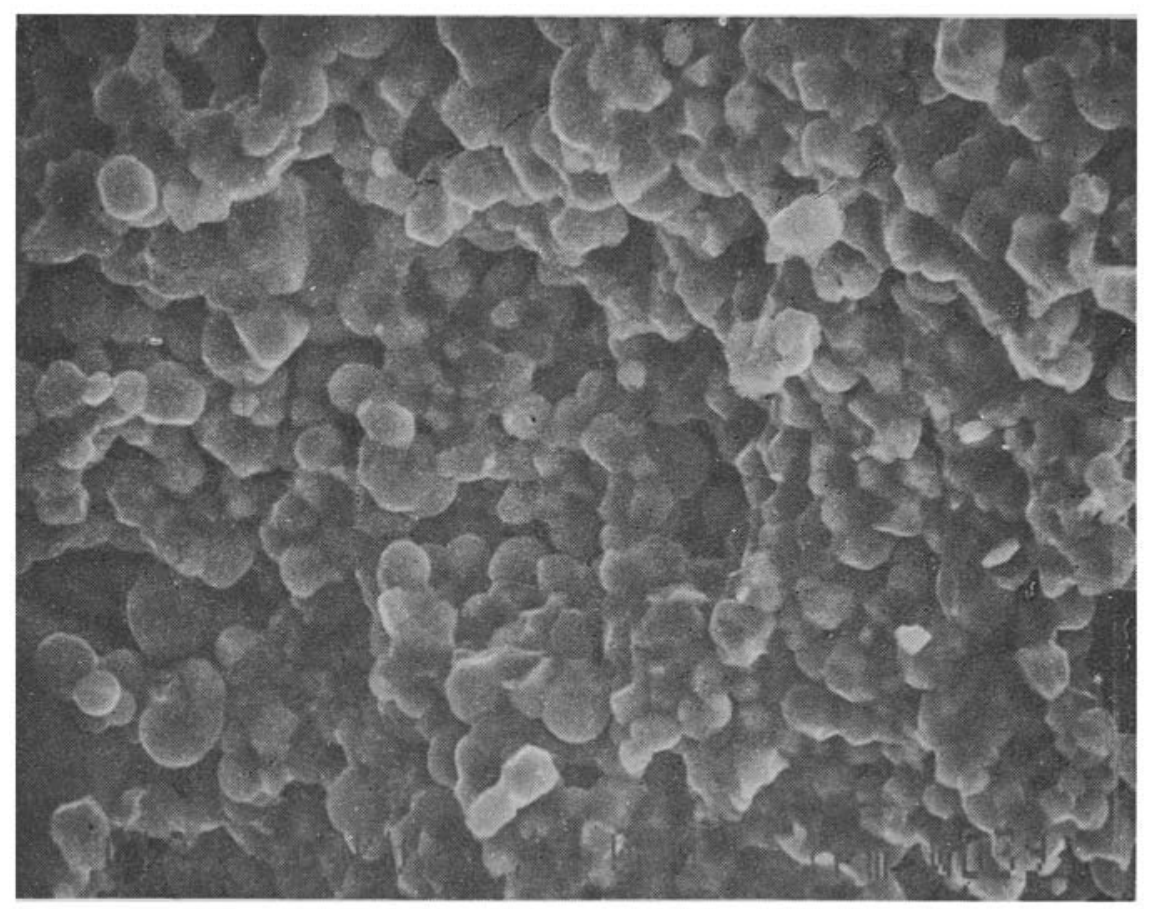

Figure 2. Fractured surface of sintered TZP-O. 


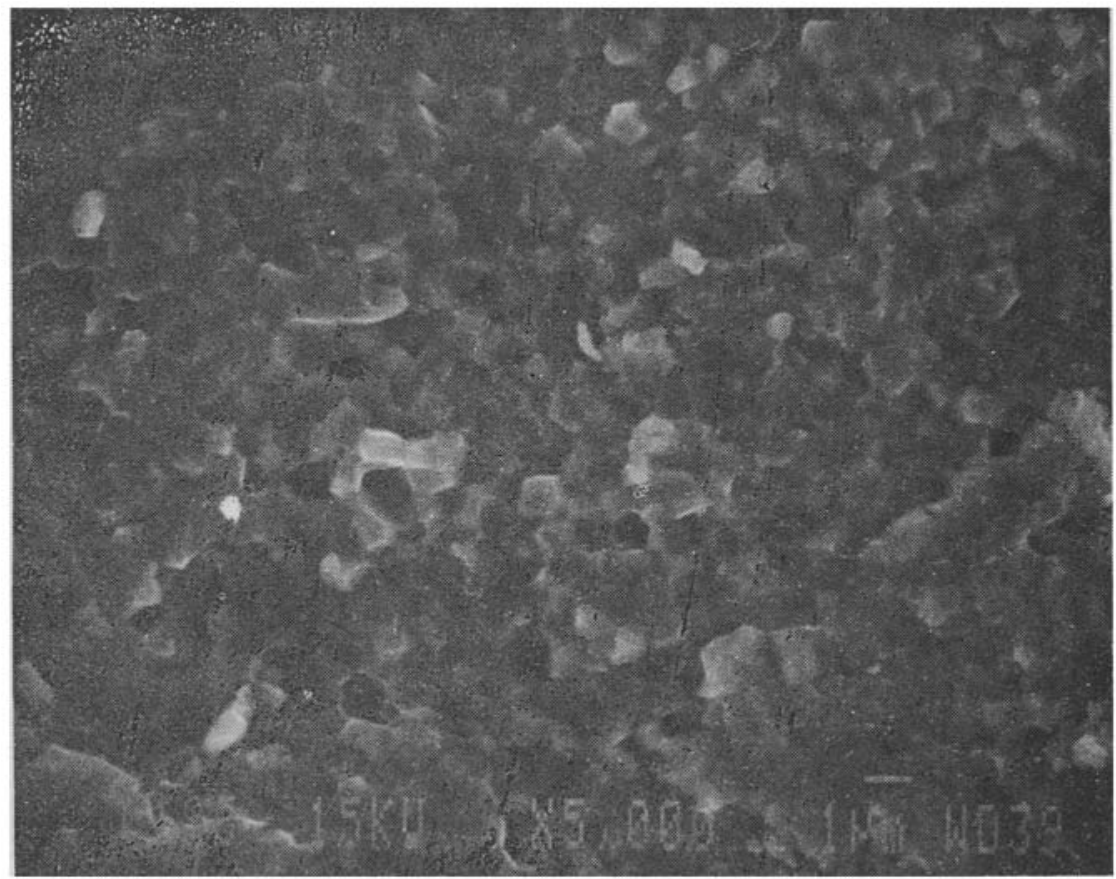

Figure 3. Fractured surface of sintered TZP-CHEM.

diffusion. Although the powder TZP-O was fine it contains $2 \%$ organic binder. Since our microwave sintering schedule was faster, it is likely that the organic binder was not removed at the preheating stage and it might have decomposed and evaporated at the rapid sintering stage, hindering complete pore elimination.

\section{Conclusions}

Y-TZP ceramic samples of different types were successfully densified from initial green density of $\sim 60 \%$ to densities close to the theoretical density by using a single-mode applicator operating in the $\mathrm{TM}_{012}$ mode at $2.45 \mathrm{GHz}$. Practical-sized samples of this material have been truly microwave-sintered without use of any binder or susceptor.

Fine and uniform microstructure in Y-TZP ceramic can be achieved by rapid sintering in a microwave cavity.

\section{Acknowledgements}

The authors wish to thank Dr Walter A Johnson, Director, Institute of Materials Processing, MTU, for his continuous encouragement and support. Thanks are due to Ruth Kramer for her assistance with the SEM work. 


\section{References}

Asmussen J, Lin H H, Manring B and Fritz R 1987 Rev. Sci. Instrum. 581477

Chabinsky I J and Eves E E 1986 Inter. Ceram. 630

Garvie R C 1984 J. Mater. Sci. Lett. 3315

Green D J 1982 J. Am. Ceram. Soc. 65610

Lange F F and Green D J 1981 in Advances in ceramics (eds) A H Heuer and L W Hobbs (Columbus, Ohio: The American Ceramic Society) Vol. 3 p. 217

Patil D S, Mutsuddy B C, Gavulic J and Dahimene M 1991 a Ceramic transactions (eds) D E Clark, F D Gac and W H Sutton (Columbus, Ohio: The American Ceramic Society) Vol. 21 p. 565

Patil D S, Mutsuddy B C, Gavulic J and Dahimene M 1991b Ceramic transactions (eds) D E Clark, F D Gac and W H Sutton (Columbus, Ohio: The American Ceramic Socity) Vol. 21 p. 565

Porter D L, Evans A G and Heuer A H 1979 Acta Metall. 271649

Sutton W H 1989 Ceram. Bull. 68376

Tinga W R 1986 Mater. Res. Soc. Symp. Proc. 60105

Yamamoto O, Takeda Y, Kanno R and Kohno K 1990 J. Mater. Sci. 252805 